\title{
Charm Production in DIS at $\mathrm{H1}$
}

\author{
Katerina Lipka ${ }^{1}$ for the H1 Collaboration \\ University of Hamburg - Institute of Experimental Physics \\ Luruper Chaussee 149, 22761 Hamburg - Germany
}

\begin{abstract}
Recent results on $D^{*}$ meson production in deep inelastic scattering at HERA are presented [1]. The data were taken with the H1 detector in the years 2004 to 2006 and correspond to an integrated luminosity of $222 \mathrm{pb}^{-1}$. The analysis covers the kinematic region $5<Q^{2}<100 \mathrm{GeV}^{2}$ and $0.05<y<0.6$. The visible range for the $D^{*}$ meson is restricted to $p_{T}\left(D^{*}\right)>1.5 \mathrm{GeV}$ and $\left|\eta\left(D^{*}\right)\right|<1.5$ where about $10,000 D^{*}$ mesons are reconstructed. Single and double differential inclusive cross sections of $D^{*}$ meson production are compared to two LO Monte-Carlo simulations and a Next-to-Leading Order calculation in the massive scheme.
\end{abstract}

\section{Charm production in ep scattering at HERA}

In deep inelastic electron-proton scattering at HERA charm quarks are produced predominantly in the photon-gluon fusion process. The mass of the charm quark provides an additional hard scale which makes the calculations in the framework of pQCD possible. Since the gluon is always directly involved in the boson-gluon fusion process, charm production becomes an important tool for the determination of the gluon density in the proton. Recent upgrades in accelerator performance, detector hardware and event reconstruction allows high precision measurements of charm production cross sections.

Two Monte-Carlo simulations and a Next-to-Leading Order (NLO) calculation are compared to the measured cross sections. Both Monte-Carlo simulations RAPGAP [2] and CASCADE [3] are using the Leading Order Matrix element. Higher order corrections are approximated by parton showers. The parton evolution in the RAPGAP Monte-Carlo is realised in the collinear approximation according to the DGLAP [4] equations. The CASCADE Monte-Carlo uses the unintegrated gluon density and the parton evolution is done according to the CCFM [5] equations.

The NLO calculation HVQDIS [6] is realised in the fixed flavour number scheme using 3 active flavours in the proton. Charm quarks are produced dynamically and are treated as massive. The value of the charm mass is varied between 1.3 and $1.5 \mathrm{GeV}$. The parton densities CTEQ5F3 are used. Renormalisation and factorisation scales are taken to be $\mu_{r}=\mu_{f}=\mu=\sqrt{\left(Q^{2}+4 m_{c}^{2}\right)}$ and are varied simultaneously from $0.5 \mu$ to $2 \mu$. The charm fragmentation is performed in the lab frame according to the Peterson [7] parametrisation with the value of $\epsilon=0.045$.

\section{Charm tagging via $D^{*}$ meson production at $\mathrm{H} 1$}

In this analysis $D^{* \pm}$ mesons are used to tag the charm production in deep inelastic scattering. The $D^{*}$ mesons are reconstructed using the decay chain $D^{*} \rightarrow D^{0}+\pi_{\text {slow }} \rightarrow K+\pi+\pi_{\text {slow }}$.

The data presented here [1] were collected with the H1 detector at HERA in the years 2004-2006. During this period HERA operated with $27.5 \mathrm{GeV}$ electrons $^{\mathrm{a}}$ and $920 \mathrm{GeV}$

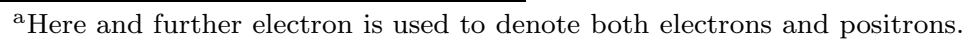


protons at a center-of-mass energy of $\sqrt{(s)}=318 \mathrm{GeV}$. The data sample used for this analysis amounts to an integrated luminosity $L=222 \mathrm{pb}^{-1}$.

The detailed description of the $\mathrm{H} 1$ detector can be found elsewhere [8]. The scattered electron is registered in a lead-scintillating fibre calorimeter, situated in the backward region of the $\mathrm{H} 1$ detector. Charged particles emerging from the interaction region are measured in the central tracking detector, the major part of which consists of two cylindrical drift chambers. The analysis covers the kinematic region $5<$ $Q^{2}<100 \mathrm{GeV}^{2}$ and $0.05<y<0.6$, where $Q^{2}$ is the four-momentum-transfer squared and $y$ is the inelasticity. The decay products of $D^{*}$ mesons are measured in the central tracking detector of H1. The range of the transverse momentum and the pseudorapidity is restricted to $1.5<p_{T}\left(D^{*}\right)<14 \mathrm{GeV}$ and $\left|\eta\left(D^{*}\right)\right|<1.5$, with $\eta=-\ln \tan (\theta / 2)$.

The signal is extracted using the mass difference distribution $\Delta m=m_{K \pi \pi}-m_{K \pi}$ of the $D^{*}$ candidates and the wrong charge combinations $\left(K^{ \pm} \pi^{ \pm}\right) \pi^{\mp}$. The wrong charge combinations provide a good description of the shape of the uncorrelated background. In Fig. 1 the $\Delta m$ distribution is shown for the $D^{*}$ candidates and the wrong charge background.

The number of $D^{*}$ mesons is determined

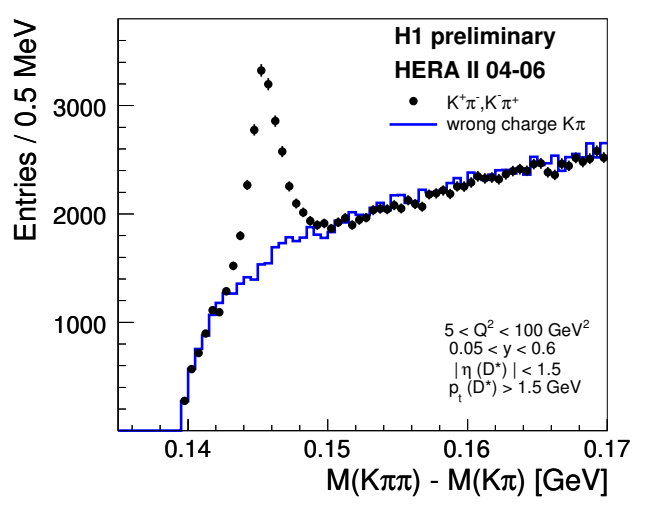

Figure 1: Mass difference distribution of $D^{*}$ candidates (closed symbols) and wrong charge background (solid line) from the simultaneous fit to the $\Delta m$ distribution of the $D^{*}$ candidates and the wrong charge combinations. The Crystall Ball fit function [9] is used to describe the shape of the signal. From a fit a total of $10000 D^{*}$ mesons is obtained. The contribution of reflections from the decays of $D^{*}$ other than the investigated one is estimated to be $4 \%$ and is taken into account.

\section{Cross section measurement}

The cross section of $D^{*}$ production is calculated from the number of $D^{*}$ mesons using the RAPGAP Monte-Carlo simulation to correct the data for the detector acceptance and efficiency. The total $D^{*}$ cross section of $4.23 \mathrm{nb} \pm 0.09_{\text {stat }} \pm 0.37_{\text {syst }}$ is obtained. The track reconstruction efficiency contributes most into the systematic uncertainty. Single differential cross sections of $D^{*}$ meson production are shown as functions of DIS kinematics in Fig. 2 in comparison to the two LO Monte-Carlo models and the NLO calculation. Both Monte-Carlo models, based on the DGLAP and the CCFM evolution equations, and the NLO calculation describe the data equally well. The cross section as a function of $D^{*}$ meson kinematics is shown in Fig. 3. Both RAPGAP and CASCADE models describe the pseudorapidity distribution well, while the NLO calculation underestimates the cross section in the forward region. The $D^{*}$ cross section as a function of the inelasticity of the $D^{*}$ meson, which is defined as $z_{D}=\left(E\left(D^{*}\right)-p_{z}\left(D^{*}\right)\right) / 2 y E_{e}$, where $E_{e}$ is the electron beam energy, is shown in the right panel of Fig. 3. The inelasticity $z_{D}$ represents the fraction of the photon energy carried by the $D^{*}$ meson. This distribution is described poorly by the models. These features have been observed with the previous measurements [10] and become more prominent with 
the higher statistics of the recent data.
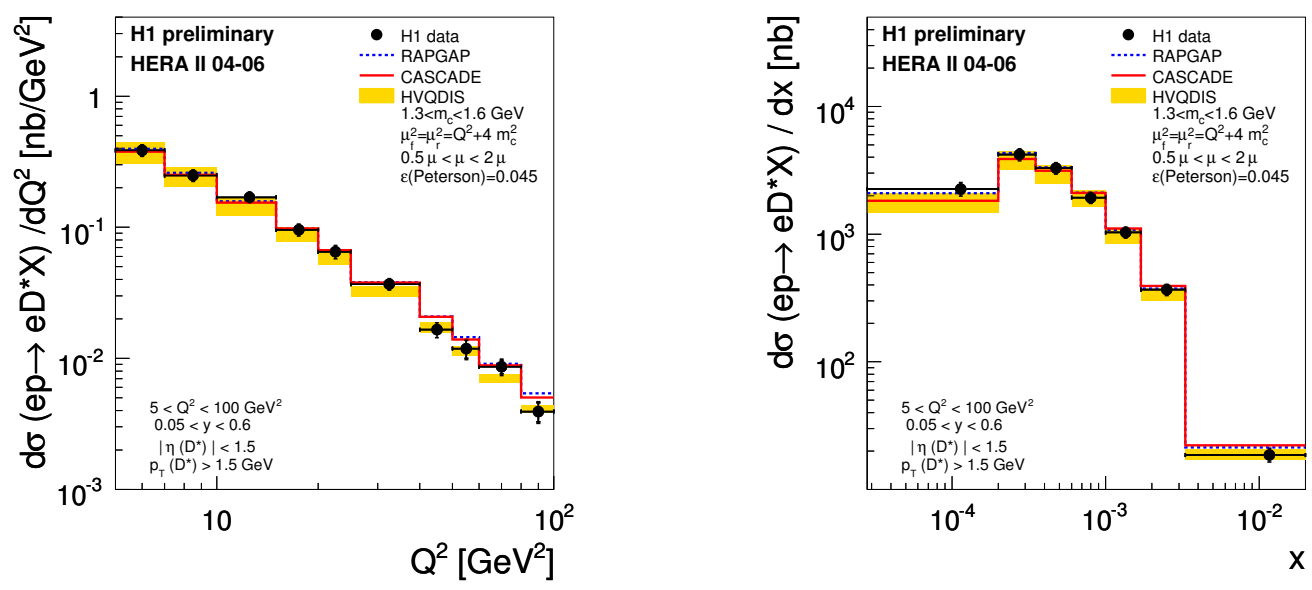

Figure 2: Differential cross section of $D^{*}$ production as a function of $Q^{2}$ (left) and $x$ (right). The data (closed symbols) are compared to the RAPGAP (dashed line) and CASCADE (solid line) Monte-Carlo simulations and the NLO calculation (shaded band).
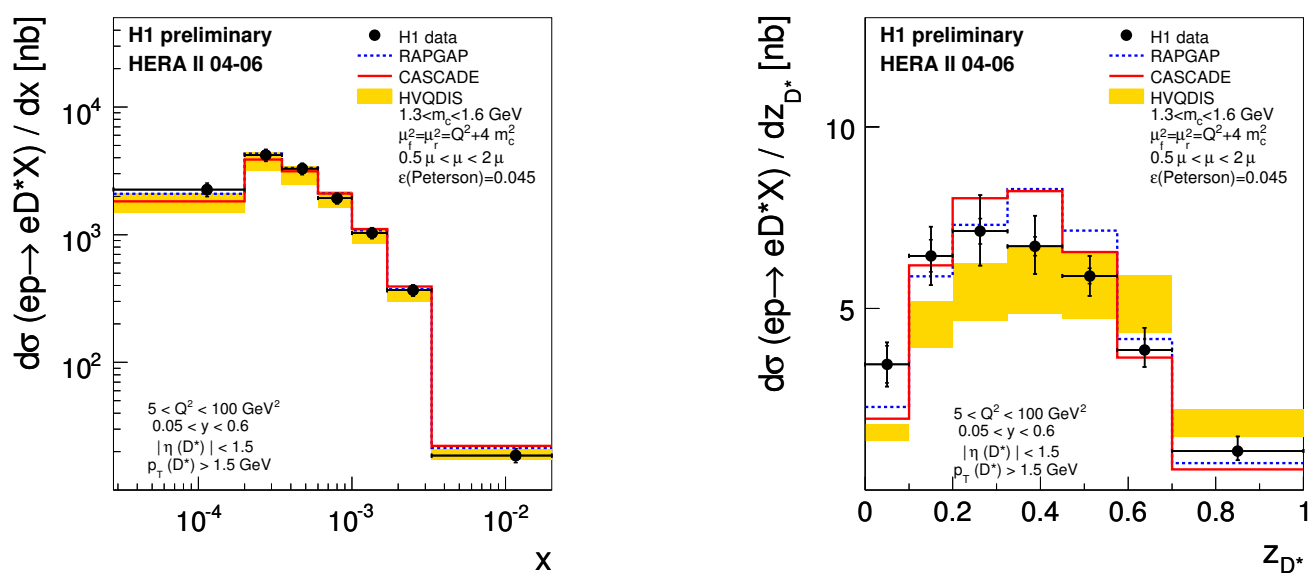

Figure 3: Differential cross section of $D^{*}$ production as a function of $\eta\left(D^{*}\right)$ (left) and inelasticity of the $D^{*}, z_{D}$, (right). The data (closed symbols) are compared to the LO Monte-Carlo simulations (solid line) and the NLO calculation (shaded band).

The high statistics allows also to make more differential studies. In Fig. 4 the doubledifferential cross section of the $D^{*}$ production are shown as a function of pseudorapidity $\eta\left(D^{*}\right)$ and transverse momentum $p_{T}\left(D^{*}\right)$. In general, the NLO calculation and the LO simulations are consistent with the data, however the deficits of the data description by all the models is visible. Within the theoretical uncertainties due to charm mass and scale variation 
HVQDIS describes the data well, except of the lowest $p_{T}\left(D^{*}\right)$ bin. The CASCADE simulation overestimates the data at high $p_{T}\left(D^{*}\right)$, which indicates that the gluon density used in CASCADE is too large at high gluon momenta. Similar feature was observed in the previous measurement [10].

\section{Conclusions and outlook}

Recent $\mathrm{H} 1$ measurements of $D^{*}$ meson production cross section are presented. About a half of the full statistics collected by $\mathrm{H} 1$ is analysed. The kinematical range for this analysis is given by $5<Q^{2}<100 \mathrm{GeV}^{2}, 0.05<y<0.6$, and the visible $D^{*}$ range is defined by $p_{T}\left(D^{*}\right)>1.5$ $\mathrm{GeV},\left|\eta\left(D^{*}\right)\right|<1.5$. The data is described by both the LO Monte-Carlo simulations. The NLO calculation is consistent with the data within theoretical uncertainties due to the variation of renormalisation and factorisation scales and the charm mass. With the increasing precision of the measurement the deficits of the models to describe the data become visible but still more precise data are needed to differentiate between the theoretical approaches. The whole statistics of the data collected by $\mathrm{H} 1$ is been analysed and both statistic and systematic precision of the data will improve significantly.
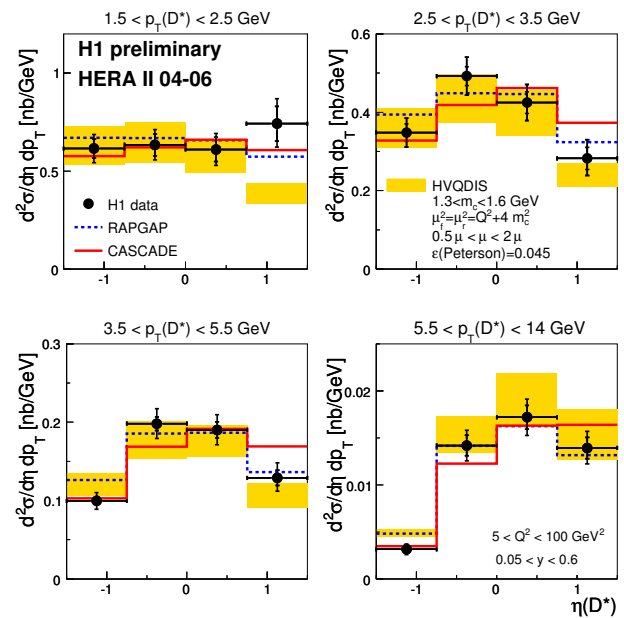

Figure 4: Double-differential cross section of $D^{*}$ production as a function of $\eta\left(D^{*}\right)$ and $p_{T}\left(D^{*}\right)$. The data (closed symbols) are compared to the RAPGAP (dashed line) and CASCADE (solid line) MonteCarlo simulations and the NLO calculation (shaded band).

\section{References}

[1] Slides: http://indico.cern. ch/contributionDisplay.py? contribId=190\&sessionId=5\&conf Id=9499

[2] H. Jung, Comput. Phys. Commun. 86147 (1995).

[3] H. Jung, Comput. Phys. Commun. 143100 (2002).

[4] V. Gribov, L. Lipatov, Yad. Fiz. 15781 (1972); G. Altarelli, G. Parsi, Nucl. Phys. B126 298 (1977) Y. Dokshitzer, Zh. Eksp. Teor. Fiz. 731216 (1977).

[5] M. Ciafaloni, Nucl. Phys. B.296 49 (1988); S. Catani, F. Fiorani, G. Marchesini, Nucl. Phys. B336 18 (1990).

[6] B. Harris,J. Smith, Nucl. Phys. B452 109 (1995).

[7] C. Peterson et al. Phys.Rev. D 572806 (1998)

[8] I. Abe et al., Nucl. Instrum. Meth. A 386310 (1997)

[9] J. E. Gaiser, Charmonium Spectroscopy from readiative Decays of the J/ $\Psi$ and $\Psi^{\prime \star}, \mathrm{Ph} . \mathrm{D}$. Thesis,Stanford University (1982)

[10] A. Aktas [H1 Collaboration] et al. hep-ex/0701023 